OPEN ACCESS

Edited by:

Paola Patrignani,

University of Studies G. d'Annunzio

Chieti and Pescara, Italy

Reviewed by:

Rania Harati,

University of Sharjah, United Arab

Emirates

Annalisa Contursi,

University of Studies G. d'Annunzio

Chieti and Pescara, Italy

*Correspondence:

Konstantinos Stamatakis

konstantinos.stamatakis@uam.es

Manuel Fresno

mfresno@cbm.csic.es

Specialty section: This article was submitted to Inflammation Pharmacology,

a section of the journal

Frontiers in Pharmacology

Received: 31 October 2021 Accepted: 21 December 2021 Published: 28 January 2022

Citation:

Stamatakis $K$, Torres-Gérica $P$, Jiménez-Segovia A, Ramos-Muñoz E, Crespo-Toro L, Fuentes P, Toribio ML,

Callejas-Hernández F, Carrato $A$,

García Bermejo ML and Fresno M (2022) Cyclooxygenase 2 Effector Genes as Potential InflammationRelated Biomarkers for Colorectal

Cancer Circulating Tumor Cells

Detection by Liquid Biopsy.

Front. Pharmacol. 12:806395.

doi: 10.3389/fphar.2021.806395

\section{Cyclooxygenase 2 Effector Genes as Potential Inflammation-Related Biomarkers for Colorectal Cancer Circulating Tumor Cells Detection by Liquid Biopsy}

\author{
Konstantinos Stamatakis ${ }^{1,2 *}$, Patricia Torres-Gérica ${ }^{1,2}$, Alba Jiménez-Segovia ${ }^{1,2}$, \\ Edurne Ramos-Muñoz ${ }^{3}$, Lorena Crespo-Toro ${ }^{3}$, Patricia Fuentes ${ }^{1}$, María L. Toribio ${ }^{1}$, \\ Francisco Callejas-Hernández ${ }^{1,2}$, Alfredo Carrato ${ }^{3}$, María Laura García Bermejo ${ }^{3}$ and \\ Manuel Fresno ${ }^{1,2 *}$ \\ ${ }^{1}$ Centro de Biología Molecular Severo Ochoa, UAM/CSIC, Madrid, Spain, ${ }^{2}$ Department of Molecular Biology, Universidad \\ Autónoma de Madrid, Madrid, Spain, ${ }^{3}$ Instituto Ramón y Cajal de Investigación Sanitaria, Madrid, Spain
}

Cyclooxygenase 2 (COX2) has been implicated in cancer development and metastasis. We have identified several COX2-regulated inflammation-related genes in human colorectal cancer cells and shown that some of them play important roles in tumor progression. In this work, we have studied the COX2-regulated genes in the mouse colorectal cancer cell line CT26, to find that many are also regulated by COX2 overexpression. On the other hand, we generated a CT26 cell line expressing Gfp and Luciferase, to study tumor growth and metastasis in immunocompetent Balb/c mice. We then collected solid tissue, and blood samples, from healthy and tumor-bearing mice. Using the Parsortix ${ }^{\circledR}$ cell separation system and taking advantage of the fact that the tumor cells expressed Gfp, we were able to identify circulating tumor cells (CTCs) in some of the mice. We compared the mRNA expression levels of Ptgs2 and effector genes in the samples obtained from tumor-bearing or healthy mice, namely, tumor or healthy colon, Ficoll purified buffy coat, and Parsortix-isolated cells to find different patterns between healthy, tumor-bearing mice with or without CTCs. Although for genes like $\| 15$ we did not observe any difference between healthy and tumor-bearing mice in Ficoll or Parsortix samples; others, such as Egr1, Zc3h12a, Klf4, or Nfat5, allowed distinguishing for cancer or CTC presence. Gene expression analysis in Ficoll or Parsortix processed samples, after liquid biopsy, may offer valuable diagnostic and prognostic information and thus should be further studied.

Keywords: colorectal cancer, liquid biopsy, circulating tumor cells, cyclooxygenase 2-regulated genes, Parsortix system, inflammation-related genes 


\section{INTRODUCTION}

Colorectal cancer (CRC) is the second cause of cancer death in the developed countries. In the last decade, a small decrease in the death numbers caused by this type of cancer has been achieved, mainly due to prevention and early screening (Siegel et al., 2020). The recent COVID-19 outbreak has led to a drastic reduction (86\%) of early screening (Patel et al., 2020), which leads to the increase of advanced cancer cases (Aguiar et al., 2021) and the need to implement new prognostic tools and treatment strategies. Circulating tumor cell (CTC) enumeration is used as a prognostic tool in different cancer types, among them, metastatic colorectal cancer (Sastre et al., 2012; Sotelo et al., 2015). The CTCs have been found to be very heterogenous, varying in surface marker expression, which reduces the value of FDA-approved tools, such as the CellSearch system (Gervasoni et al., 2011; Raimondi et al., 2014). Taking advantage of the physical characteristics of the CTCs, rather than the specific surface or expression markers, to isolate them, the Parsortix system has been able to isolate CTCs from a great variety of tumors and patients and it has been used also in preclinical models (Kitz et al., 2018; Obermayr et al., 2018; Obermayr et al., 2019).

Cyclooxygenase 2 (COX-2) is widely accepted to be implicated in CRC progression and metastasis, as well as an important therapeutic target for treatment or prevention of this type of cancer (Doherty and Murray, 2009; Wang and DuBois, 2010). In an effort to identify the mechanisms through which COX-2 activity leads to CRC progression, we showed that its overexpression is enough to increase cancer cell aggressiveness (Stamatakis et al., 2015) through the regulation of the expression of genes we consider COX-2 activity effectors in the cancer cells (Hidalgo-Estévez et al., 2020). A recent study pointed out that COX-2 is expressed in CTCs of CRC patients and associated with the clinicopathological features of the patients (Cai et al., 2019).

We decided to study the effect of COX-2 and its effector genes in a mouse model of colorectal cancer and metastasis. We confirmed that COX-2 activity had similar effects on the COX-2-effector genes as in human CRC cells. Moreover, we studied the expression of these genes, as well as Ptgs2, the mouse gene encoding Cox-2, in tumors, peripheral blood-nucleated cell isolates, and in CTCs isolated with the Parsortix system. We found that the expression levels of this group of genes can help estimate the presence of CTCs and that this strategy could be useful to identify new molecular markers for CTCs.

\section{MATERIALS AND METHODS}

\section{Mice and Mouse Models}

All animal studies were done according to Spanish and European regulations, the Ethics Committee of Animal Experimentation (CSIC-UAM), and the Institutional Review Board of UAM. Sixweek-old female Swiss Nude and Balb/c mice were obtained from Janvier labs. For subcutaneous inoculation, $10^{6}$ cells were injected under the skin of the left flank of the mice. Orthotopic inoculation in the cecum wall of 50,000 cells was done according to the method described by Tseng et al. (2007). Tumor growth was monitored by bioluminescence, using an IVIS Lumina system (Perkin Elmer), as described before (Jiménez-Segovia et al., 2019). Fifteen mice were inoculated, 11 of which developed tumors. At the end of the experiment, mice were sacrificed by $\mathrm{CO}_{2}$ inhalation, blood was collected through heart puncture, and tumors were excised for further processing (macroscopic separation of tumors and RNA extraction). From the 11 blood samples, four were discarded due to extensive clotting (although all animals were injected intraperitoneally with heparin to avoid this). Samples from five healthy mice were used as controls.

\section{Cell Culture}

Colorectal carcinoma cell line CT26 was obtained from the ATCC and maintained according to the distributor's instructions. Human COX2 overexpression was achieved after transduction with a lentiviral vector and antibiotic selection, as described before Stamatakis et al. (2015). The CT26-Luc-Gfp cell line was generated by transducing CT26 cells with lentiviral particles generated with the pHRSIN-Luc-IRES-Gfp vector, originally described in Garaulet et al. (2013), carrying the firefly luciferase and Gfp cDNAs separated by an IRES. Gfppositive cells were sorted with a FACSAria Fusion (BD Biosciences), and light-emitting clones were selected, adding D-luciferin to the medium and detecting light emission with a BMG Biosciences FluoStar Optima plate reader. After inoculation and tumor growth in nude mice, tumors were excised and tumor cells cultured and sorted for Gfp positive cells. These cells were used for further experiments in Balb/c mice.

\section{Reagents and Materials}

All reagents were purchased from Sigma Aldrich, unless stated otherwise. Oligonucleotides were synthetized by Sigma Aldrich, according to the following sequences (gene symbol, forward primer, reverse primer): Ptgs $2,5^{\prime}$ gatgctcttccgagctgtgc, $5^{\prime}$ gga ttggacagcaaccatttg; Ptges, $5^{\prime}$ gtgatctcctggctgcaaatc, $5^{\prime}$ cctggacagtgc tttgctctg; Dusp10, 5' cctgtcgtctaaaggagatgga, 5' cagatggtagagggc tcgc; PMEPA1, 5' gaccatcttcgacagtgacct, gtagcaggtggcgctgatg; KLF4, $5^{\prime}$ atggtcaagttcccagcaag, $5^{\prime}$ tttctgtttgtctcttgaactcttc; Tacstd2: $5^{\prime}$ cgggcaaatacaaaaaggtg, $5^{\prime}$ acaagctaggttcgcttctca; Zc3h12a, $5^{\prime}$ tcatcgacggaagcaatgt, $5^{\prime}$ cctcgctccagaaaccag; Nfat5, $5^{\prime}$ tcagacaagcggtggtga, $5^{\prime}$ agggagctgaagaagcatca; Ptgf $r, 5^{\prime}$ tgcaat gttggccattgttacg, $5^{\prime}$ ctggccataatgtgcgtctc; Egr $1,5^{\prime}$ tcacctatactggcc gcttc, $5^{\prime}$ ggttcaggccacaaagtgttg; $T g f b 1,5^{\prime}$ ccaaggtaacgccaggaattgttg ctata, $5^{\prime}$ agcggactactatgctaaagaggtcaacc; Inhba 1, $5^{\prime}$ tcctcttcatggtat tggca, $5^{\prime}$ gggagtgatccctggaaac; Il15ra, $5^{\prime}$ tgcagaagttgtttgggatg, $5^{\prime}$ tacccgcaatgaccacagaga; Il15, $5^{\prime}$ actgtcagtgtataaagtggtgtcaatatg, $5^{\prime}$ cagaggccaactggatagatgtaag; Nfkbia, 5' cgaggagtacgagcaaatgg, $5^{\prime}$ tgattgccaagtgcagga; Gadph, $5^{\prime}$ tgtagaccatgtagttgaggtca, $5^{\prime}$ aggtcg gtgtgaacggatttg.

\section{Isolation of Blood-Nucleated Cells (Ficoll Samples)}

Mouse blood was collected in EDTA-treated tubes, to avoid coagulation; $1 \mathrm{ml}$ of Ficoll Paque (FisherSci) was placed beneath the blood in the tube and centrifuged at 1,500 rpm with for $30 \mathrm{~min}$ according to the manufacturer's instructions. 
The upper, clear phase containing all nucleated cells was collected, diluted in PBS, and centrifuged to obtain the cell pellet.

\section{Isolation of CTCs With the Parsortix System}

Mouse blood was isolated as above, diluted 1:1 with PBS, and introduced to the Parsortix system (Angle PLC, Surray, United Kingdom), according to the manufacturer's instructions, collecting the CTCs in a $6.5 \mu \mathrm{m}$ cassette using the Parsortix PX2_S99F protocol. Once the protocol was finished, the cassette was removed and observed under an Axiovert200 (Zeiss) fluorescence microscope with a sCMOS monochrome camera for presence of Gfp-positive cells (CTCs, since only tumor cells are Gfp-positive). To avoid cell loss or RNA degradation, cells were immediately lysed in the cassette through the flow of $300 \mu \mathrm{l}$ of isolation buffer (SPLIT RNA extraction kit), collecting all the flowthrough volume, before proceeding to RNA extraction. Parsortix system-isolated CTCs have been characterized in other studies (Gkountela et al., 2019; Szczerba et al., 2019).

\section{Gene Expression Analysis}

RNA was extracted using Trizol reagent (Thermo Fisher Scientific) for cell culture or tumor samples and with the SPLIT RNA extraction kit (Lexogen) for Parsortix cassette and Ficoll samples. The RNA was retrotranscribed with the Transcriptor First Strand cDNA Synthesis Kit (Roche Diagnostics). Quantitative PCR was performed using the GoTaq 2-Step RT-PCR system (Promega). Relative mRNA levels to the Gadph housekeeping gene $(\Delta \mathrm{Ct})$ and to the experimental control point $(\Delta \Delta \mathrm{Ct})$ were calculated using the $2^{-\Delta \Delta \mathrm{CT}}$ formula from the values obtained. To quantify and compare gene mRNA levels between Parsortix samples (less than 1,000 cells), Ficoll-isolated buffy coats (millions of cells), and tumor samples (millions of cells), the entire eluate of the SPLIT RNA extraction kit was used for the first type of samples, while 50 ng RNA was used for the rest, for retrotranscription. Preamplification and qPCR amplification were performed using the RealTime ready cDNA Pre-Amp Master (Roche Diagnostics) with the RealTime ready Pre-Amp Primer Pool, and the RealTime ready custom panel (Roche Diagnostics) according to the manufacturer's instructions, using a LightCycler 480 (Roche Diagnostics). mRNA levels of each gene were expressed as $\Delta \mathrm{Ct}$, the difference of the crossing point $(\mathrm{Ct})$ of the gene with the $\mathrm{Ct}$ of the housekeeping Gadph.

\section{Statistical Analysis and Principal Component Analysis}

Statistical analysis was performed with the Graphpad Prism software. Depending on the data compared, unpaired $t$-test, Wilcoxon test, or Mann-Whitney $U$ test was performed. Significance is indicated in the figure legends.

Principal component analysis (PCA) (Mardia et al., 1979; Becker et al., 1988; Venables and Ripley, 2002) was conducted in RStudio 2021.9.0.351 (R Core Team, 2021 https://www.rstudio. com/) using prcomp command, and ggbiplot package (Vincent Q. $\mathrm{Vu} 2011 \mathrm{https} / /$ github.com/vqv/ggbiplot) was used for data visualization.

\section{RESULTS}

\section{COX2-Regulated Genes in CT26 Cells}

We have identified in previous works (Stamatakis et al., 2015; Hidalgo-Estévez et al., 2020) genes regulated by cyclooxygenase activity in HT29 human colon cancer cells. Among the upregulated genes were PTGES, DUSP10, PMEPA1, KLF4, TACSTD2, ZC3H12A, NFAT5, PTGFR, EGR1, TGFB1, INHBA, IL15RA, IL15, and NFKBIA. In this work, we overexpressed COX2 in the mouse colon cancer cell line CT26, to test if these genes were also upregulated. CT26 cells in culture express low levels of Ptgs2, which increased when we overexpressed the human COX2, probably through a positive feedback loop. Although there was a tendency for many of the mouse homologs of the mentioned genes to be upregulated, we found that this was significant only for Ptges, Dusp10, Inhba, Il15, and Nfkbia (Figure 1). COX2 overexpression has been associated with colorectal cancer progression and metastasis, it is upregulated in tumors vs. normal colon tissue and we have shown that PTGS2 (that encodes COX2) is also up-regulated in human xenografts in nude mice (Stamatakis et al., 2015). Using the CT26-Balb/c syngeneic, orthotopic mouse model, we tested if the mRNA levels of PTGS2 and the abovementioned effector genes varied between normal colon and tumor tissue. As it can be seen in Figure 2, Ptgs2 levels are significantly higher in tumor tissue than normal colon. Similar results were obtained for Ptges, Dusp10, and Inhba1, suggesting that the Ptgs2 upregulation could be responsible for their increased mRNA levels. On the contrary, Pmepal, Klf4, Il15ra, Il15, and Nfkbia were significantly decreased indicating that other factors may be more important than the cyclooxygenase activity for their regulation in vivo. We also checked Ptgfr expression levels to find that both in culture as in vivo, it had an inverse correlation with Ptgs2 expression levels. Moreover, when comparing data from our mouse model and from the TCGA colon cancer cohort analyzed using the UCSC Xena browser (https://xenabrowser.net/), the direction in the gene expression change, between normal tissue and tumor, was similar in both human and mouse for Ptgs2, Ptges, Dusp10, Klf4, Ptgfr, Egr1, Inhba1, Il15, and Nfkbia (not shown). The rest of the genes had a different behavior when comparing colon and tumor tissue, suggesting there are differences in gene regulation and in their role in cancer progression between the two species.

\section{The CT26-Balb/C Orthotopic Colon Cancer Model Produces CTCs}

$\mathrm{Balb} / \mathrm{c}$ mice inoculated with CT26 cells rapidly produce tumors in all the length of the large intestine, even though cells were injected in the cecum wall (not shown). This could be due to cell shedding in the peritoneal cavity and colonizing the rest of the intestinal tube, or due to metastasis through the blood stream. To test the second hypothesis, we decided to search for CTCs derived from these tumors in the blood of the mice. Using a fluorescent and bioluminescent derivative of the cell line, CT26-Luc-Gfp, we inoculated mice orthotopically and monitored tumor growth by in vivo imaging (Figure 3A). When mice showed clear sign 


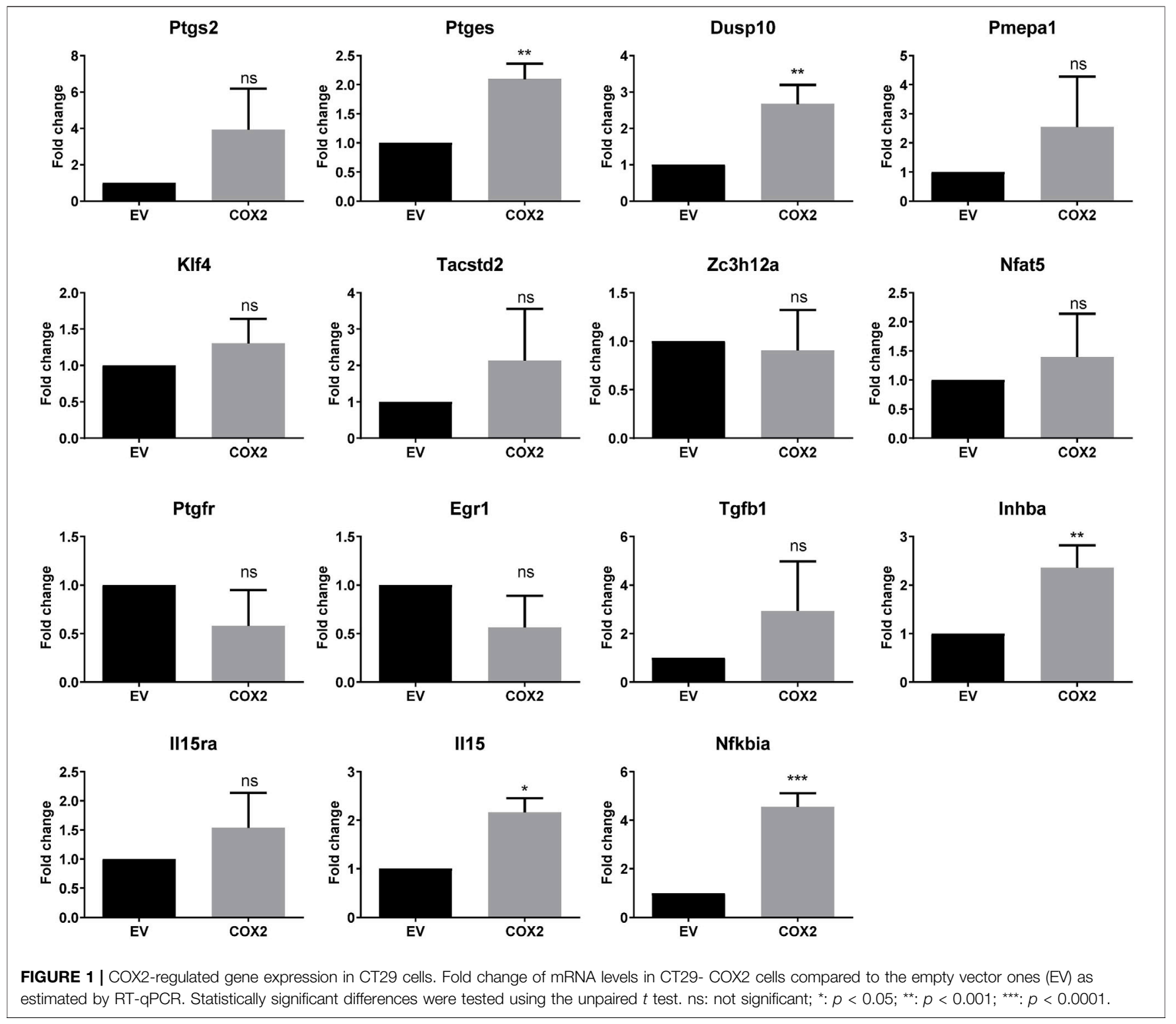

of metastatic tumor growth (bioluminescent signal expanding in the entire peritoneal cavity), they were sacrificed, and tumor growth and metastasis were confirmed (Figure 3A). At sacrifice, the blood of the mice was extracted and separated in two, for Ficoll gradient nucleated cell isolation and for Parsortix system CTC isolation. We were able to detect the CTCs in the blood of the tumor-bearing mice using fluorescent microscopy, searching for Gfp-positive cells (Figure 3B). On the other hand, we extracted RNA from the mentioned samples to compare the expression levels of our genes of interest in solid tissue, buffy coat, and Parsortix system cassette.

\section{Gene Expression Varies due to the Presence of CTCs}

As already shown in Figure 2, the expression of the COX2effector genes is different between normal colon and colon tumors. We sought to explore if the presence of tumors or tumors releasing CTCs would change the expression pattern of these genes in a detectable way, in solid tissue, in the blood or in the Parsortix system-isolated cell population. Thus, we compared, as shown in Figure 4, the mRNA levels of each gene in solid tissue (green), Ficoll isolate (black), and Parsortix isolate (red) in healthy (Healthy), tumor-bearing (wTumor) and tumorbearing with detectable CTCs ( $w C T C s)$ mice. As expected, the levels of each gene vary between sample/isolation types in each mouse. On the other hand, the tumor presence altered gene expression in a similar way to Figure 2, although it is interesting to note that the Pmepal and Klf4 expression levels were remarkably lower in tumors with detectable CTCs. In a similar way, regarding Ficoll isolates, Nfat5 and Egr1 levels were significantly lower in CTCs bearing samples while the Dusp10 levels were higher. Finally, Klf4 and Egrl levels were lower in the CTCs containing Parsortix-isolated samples, while Dusp10 and 


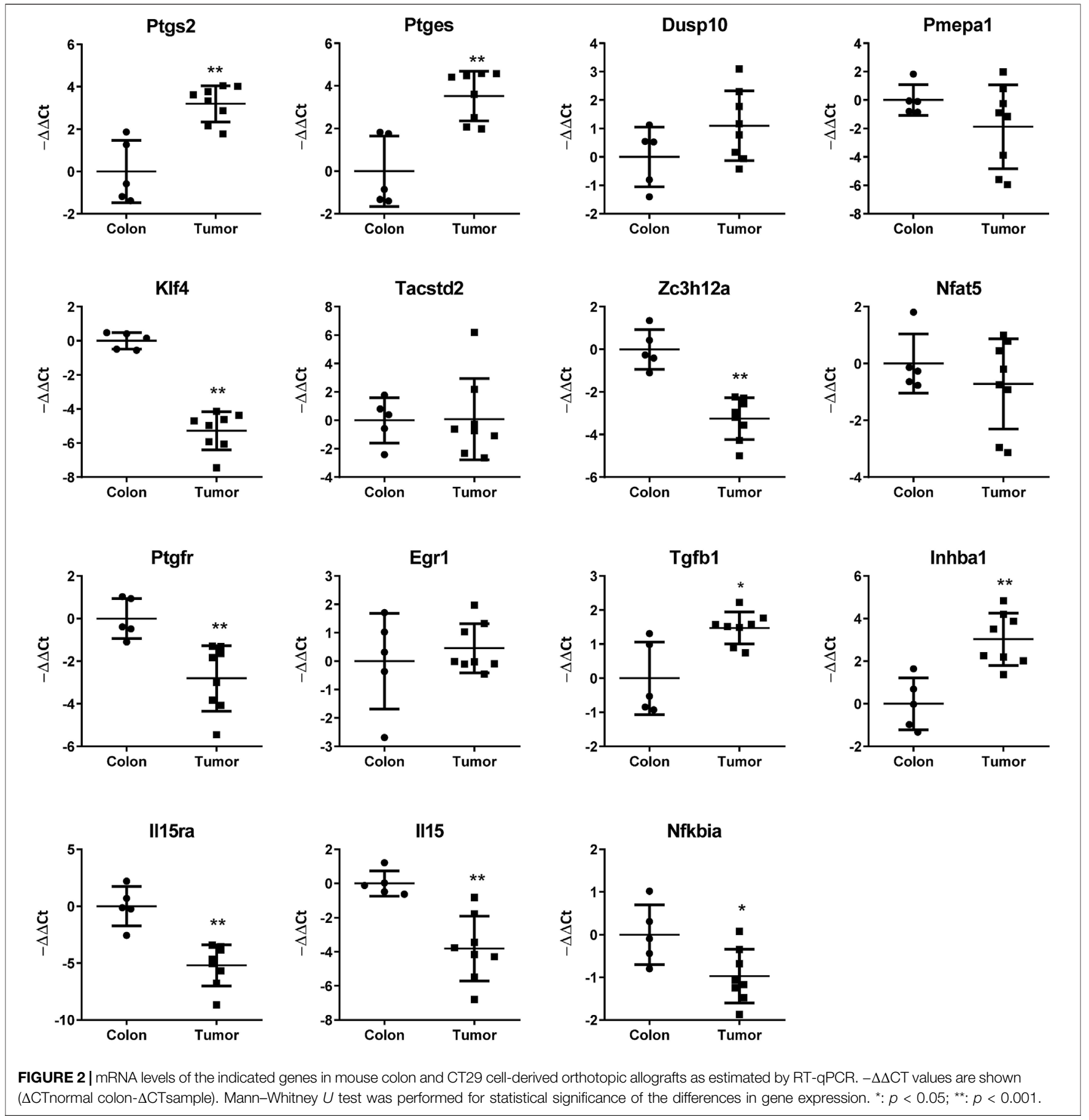

Pmepal higher levels in these samples could indicate tumor presence. These results suggest that in each isolation type, mRNA level changes can be found that may indicate the presence of tumors or tumors releasing CTCs.

To further investigate this, principal component analysis (PCA), taking into account the gene expression levels $(\Delta \mathrm{Ct})$ in the different "health conditions" and isolation method, was performed. As expected, samples are grouped by extraction method, although in the case of solid tissue, distances are greater than in the other two (Figure 5). In all extraction methods, the CTC-positive groups have lower values for PC1 (influenced mainly by Egr1, Nfkbia, Zc3h12a, Tgfb1, Ill5ra, Il15, Pmepa1, Dusp10, and Klf4, as compared with the other two, which by itself would be enough to separate the three health states in solid tissue. PC2 (driven by Ptgs2, Ptges, Inhba, Tacstd2, and Nfat5) could help distinguish between tumor and healthy, although in the Parsortix samples, healthy falls between the two tumor conditions on the PC1 axis. This confirmed that 


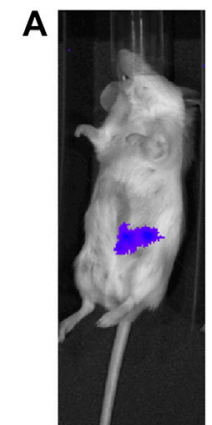

2 weeks

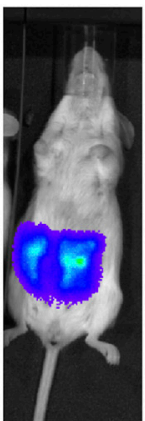

4 weeks

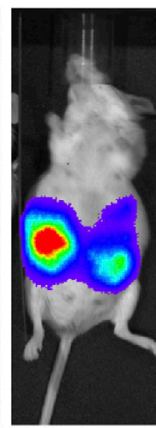

5 weeks

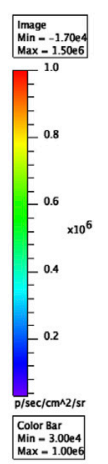

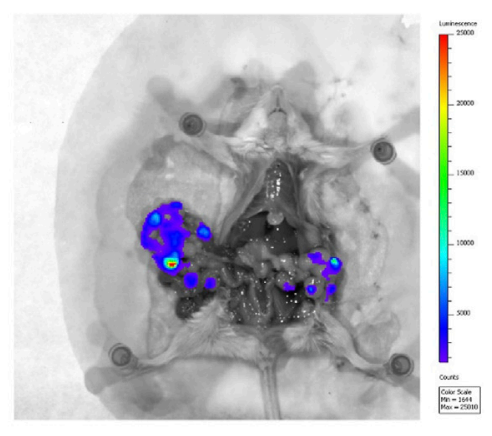

B
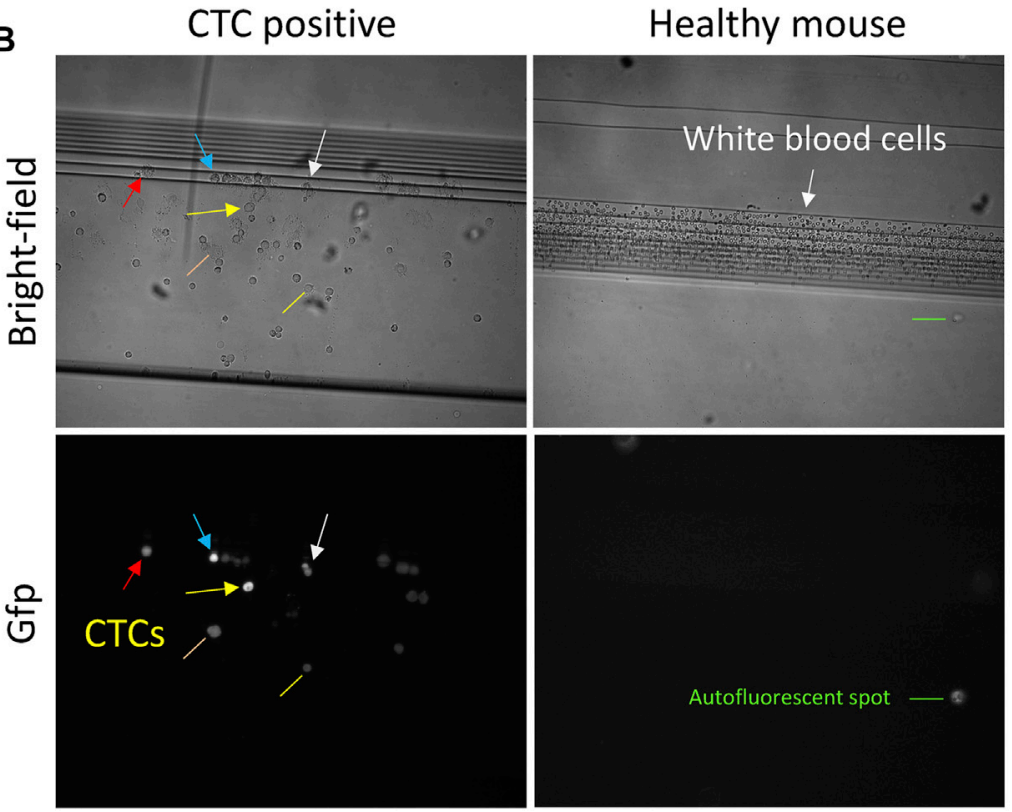

FIGURE 3 | CT26-Luc-Gfp allograft growth in Balb/c mice. (A) In vivo and ex vivo bioluminescence imaging at the indicated times and at sacrifice. (B) Bright-field (upper panels) and Gfp fluorescence (lower panels) microscopy of the cells retained in the Parsortix system cassette, from a CTC-positive and a healthy mouse. Arrows of the same color indicate the same cell in the upper and lower panel. Yellow and orange lines indicate cells with lower fluorescence intensity, possibly necrotic. Notice the absence of GFP fluorescence in the healthy mouse cassette, although numerous cells can be seen in brightfield. Green line indicates autofluorescence spot. Although in the image it might appear similar to a cell, it is fluorescent in all channels, thus it can be easily distinguished.

this group of genes could serve to distinguish between healthy, tumor-bearing, and tumor plus CTC-bearing mice.

\section{DISCUSSION}

COX2 expression and activity has been has been long considered to play a very important role in colon tumorigenesis, cancer progression, and metastasis (Kawai et al., 2002; Cathcart et al., 2011), and its inhibition has been shown to have both great preventive and treatment value (Rothwell et al., 2011; Rothwell et al., 2021). Thus, it is logical to expect a possible role for COX2 in tumor dissemination in the form of CTCs. In fact, other authors have shown a certain association of COX2 expression in CTCs and colon cancer metastasis (Cai et al., 2019). We sought to investigate the mRNA levels of Ptgs2 in our mouse model of colon cancer, CT26 cells-Balb/c mice, as well as those of the group of COX2 effector genes we identified in human colon cancer. First, we confirmed that COX2 activity can change the expression of some of the genes we have identified as COX2-target or effector genes in human cancer (Stamatakis et al., 2015; Jiménez-Martínez et al., 2019; Jiménez-Segovia et al., 2019; Hidalgo-Estévez et al., 2020), namely, Ptge2, Ptges, Dusp10, Pmepa1, Inhba, Il15, and Nfkbia. There was also a tendency for Klf4, Tacstd2, Nfat5, Tgfb1, and Il15ra to be upregulated with COX2 overexpression in CT26 cells, as it happened in HT29 human cells, but without reaching statistical significance. These results indicate that the regulation of these genes is similar in both species, regarding COX2 activity. Cell line-specific effects that could explain the differences cannot be discarded. The regulation of these genes by COX2 was also 


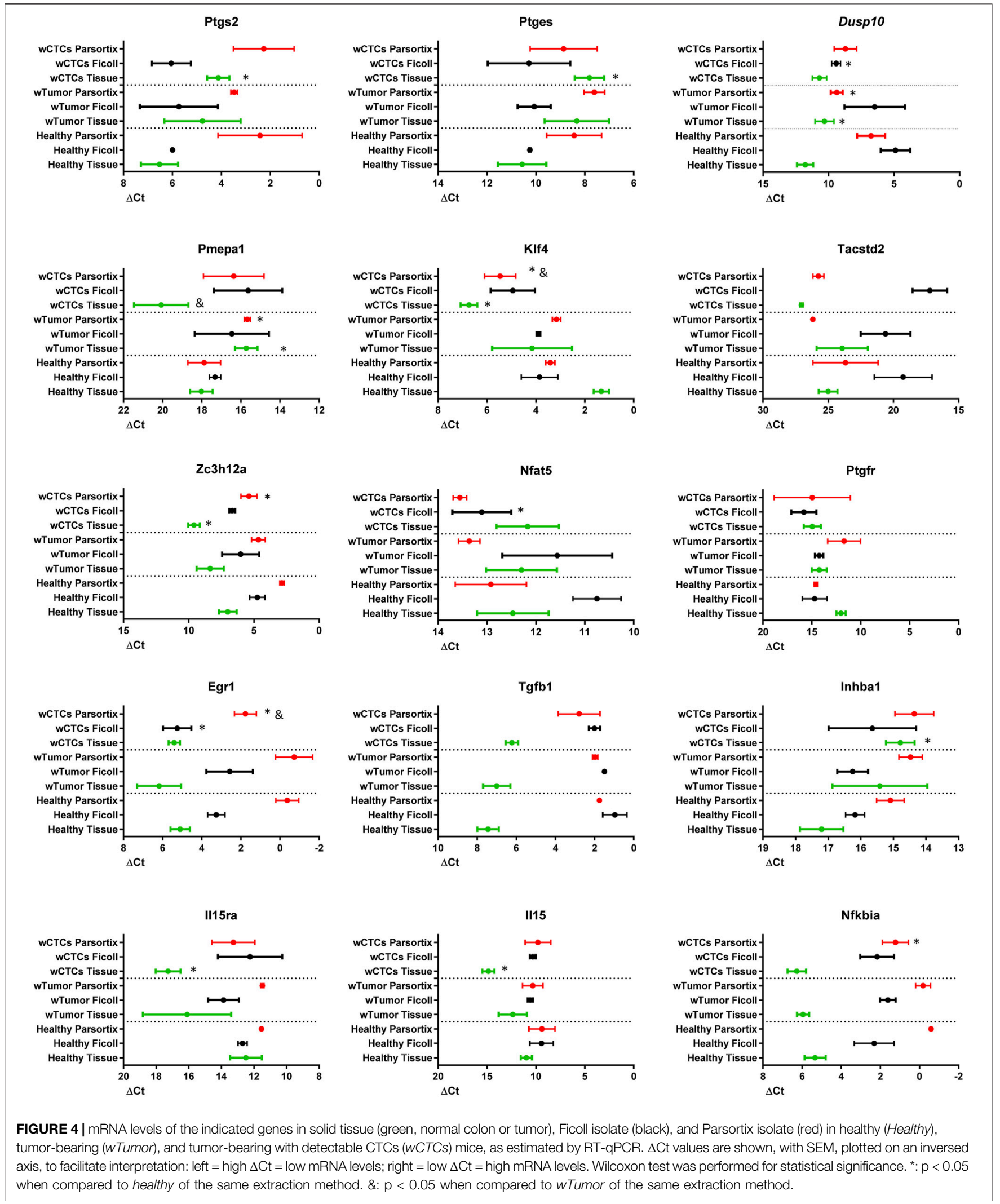




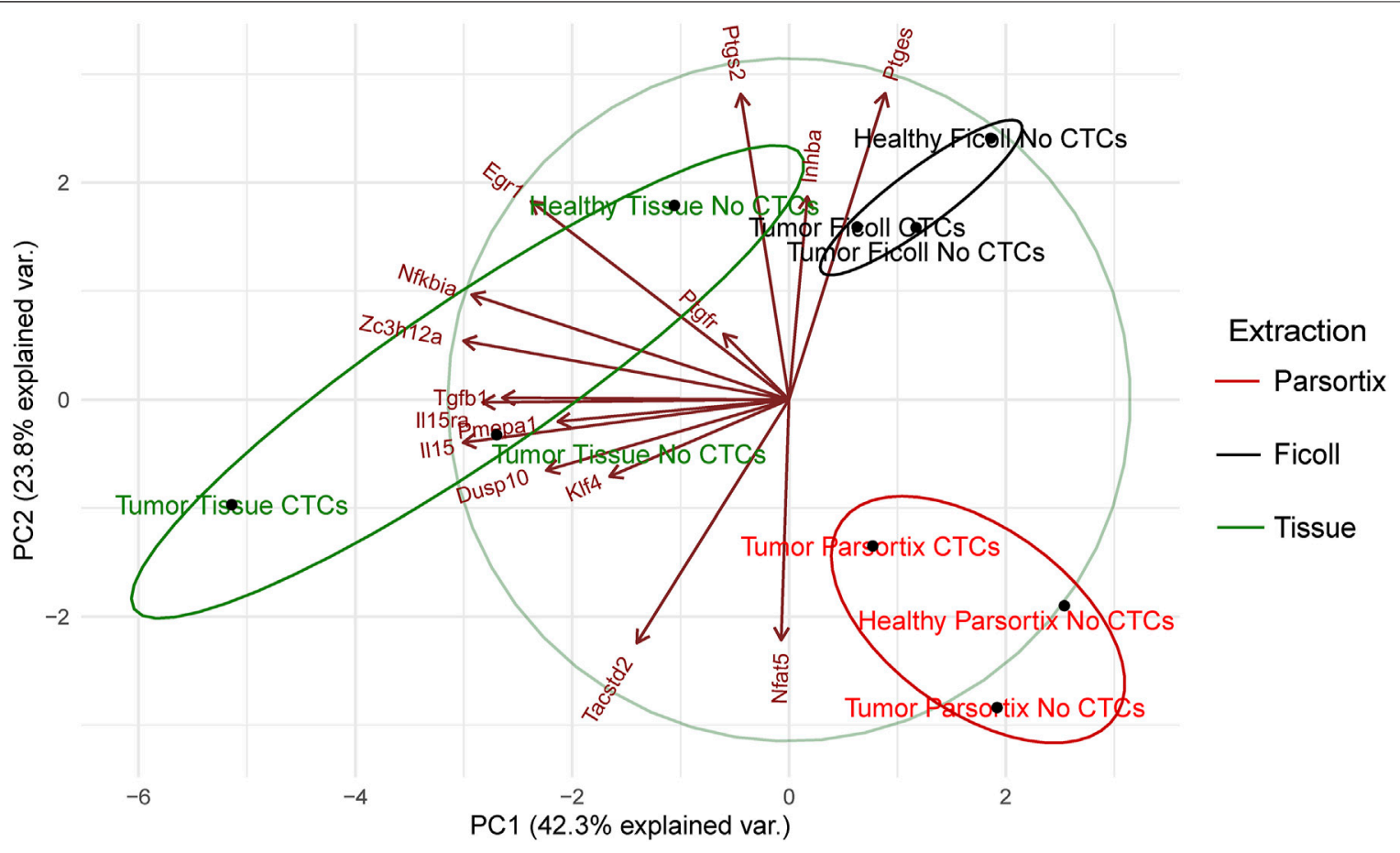

FIGURE 5 | Principal component analysis of the gene expression $(\Delta \mathrm{Ct})$ data shown in Figure 4, represented as a biplot. Sample principal component score centroids (dots) and loading of variables (vectors) are plotted. Confidence ellipses group samples by extraction type.

confirmed when analyzing gene expression in mouse allografts of the CT26 cells. Ptgfr levels were lower, both in culture as in tumors, agreeing with the human tumor data (Hidalgo-Estévez et al., 2020) and the notion that, during cancer development and progression, the arachidonate/prostanoid pathway is tuned towards $\mathrm{PGE}_{2}$ production and detection, downregulating synthases and receptors of $\mathrm{PGF}_{2 \alpha}$ and $\mathrm{PGD}_{2}$ (Cebola et al., 2015). It is important to note that our mouse allograft model is immunocompetent, simulating better the situation in patients. Immune infiltration, interaction with the tumor cells, and selection pressure may be the responsible for the differences found in the expression of Klf4, Tacstd2, Zc3h12a, Il15, Il15ra, and Nfkbia. Since the implications of the regulation of the COX2effector genes have been discussed elsewhere, we focus on the gene expression differences when we group individuals for CTCs existence.

The CT26-Luc-GFP-Balb/c singeneic cancer model allowed us to study tumor development and its interaction with the immune system. Thanks to the Parsortix System, we were able to detect CTCs specifically due to Gfp expression by these cells. Thus, when we focused to compare the mRNA levels of each gene in the different sample types, solid tissue, buffy coat, and Parsortix cassette, we were able to group animals not only in healthy and tumor-bearing groups but also for the presence of CTCs. This allowed us to observe an interesting phenomenon regarding Pmepal mRNA levels. Although they were higher in tumor tissue than in normal colon, this was not so in tumors of mice with CTCs detected, where Pmepal mRNA levels were much lower than in healthy colon. PMEPA1 has been found to be highly expressed in normal colon and most colorectal adenocarcinomas and metastases (Brunschwig et al., 2003; Zhang et al., 2019), but it would be interesting to compare tumors that actively disperse CTCs with others that do not. We have show that PMEPA1 increases cell proliferation while it induces E-cadherin expression in ovarian tumor cells (JiménezSegovia et al., 2019). While these cells are able to survive and proliferate better, they are less invasive. If this also happens in colon cancer cells, they would have to reduce PMEPA1 levels before invading blood vessels and releasing CTCs. Another possible explanation could be the effect of the tumor microenvironment which can both contribute to gene expression and shedding of CTCs. The combination of easily detectable CTCs (e.g., expressing Gfp) with the Parsortix system could greatly facilitate this kind of study, making it possible to identify the events or characteristics of tumors of the same origin (e.g., CT26-Gfp cells) to produce CTCs or not.

At the liquid biopsy level, the Egr1 and Klf4 mRNA level reduction in the Parsortix samples could be a good marker for the presence of CTCs. This reduction was also observed in the Ficoll gradient samples, indicating that the level change is probably due to a more generalized change in the expression of these genes in peripheral blood lymphocytes, the percentage of CTCs not being responsible, as it is negligible in the case of the buffy coat. Further studies comparing broad gene expression between healthy, tumor-bearing, and CTC-positive tumor-bearing individuals' liquid biopsy samples will be able to provide accurate biomarkers for CTC presence or even for cancer presence in general, especially single-cell RNAseq. This is particularly 
supported by the fact that by just analyzing a small group of genes, we were able to identify two genes of which expression levels could indicate the presence or absence of CTCs. Moreover, taking into account the mRNA levels of the 15 selected genes, we could perform a principal component analysis that clearly separated the healthy, the tumor positive-CTC-negative, and the tumor positive-CTC-positive mice. Single-cell RNAseq studies on Parsortix-isolated cells could increase the robustness of this liquid biopsy method as a diagnostic tool for colorectal cancer.

\section{DATA AVAILABILITY STATEMENT}

The original contributions presented in the study are publicly available. This data can be found here: https://xenabrowser.net/ datapages/?cohort=TCGA\%20Colon\%20Cancer\%20(COAD) \&removeHub=https:\%3A\%2F\%2Fxena.treehouse.gi.ucsc.edu\% 3A443.

\section{ETHICS STATEMENT}

The animal study was reviewed and approved by The Ethics Committee of Animal Experimentation (CSIC-UAM).

\section{AUTHOR CONTRIBUTIONS}

KS: experimental design, animal models, manuscript preparation; PT-G: sample processing, PCA; AJ-S: gene expression analysis;

\section{REFERENCES}

Aguiar, S., Riechelmann, R. P., de Mello, C. A. L., da Silva, J. C. F., Diogenes, I. D. C., Andrade, M. S., et al. (2021). Impact of COVID-19 on Colorectal Cancer Presentation. Br. J. Surg. 108, e81-e82. doi:10.1093/bjs/znaa124

Becker, R. A., Chambers, J. M., and Wilks, A. R. (1988). The New S Language: A Programming Environment for Data Analysis and Graphics. Wadsworth \& Brooks/Cole.

Brunschwig, E. B., Wilson, K., Mack, D., Dawson, D., Lawrence, E., Willson, J. K., et al. (2003). PMEPA1, a Transforming Growth Factor-Beta-Induced Marker of Terminal Colonocyte Differentiation Whose Expression Is Maintained in Primary and Metastatic colon Cancer. Cancer Res. 63, $1568-1575$.

Cai, J., Huang, L., Huang, J., Kang, L., Lin, H., Huang, P., et al. (2019). Associations between the Cyclooxygenase-2 Expression in Circulating Tumor Cells and the Clinicopathological Features of Patients with Colorectal Cancer. J. Cel Biochem 120, 4935-4941. doi:10.1002/jcb.27768

Cathcart, M. C., Lysaght, J., and Pidgeon, G. P. (2011). Eicosanoid Signalling Pathways in the Development and Progression of Colorectal Cancer: Novel Approaches for Prevention/intervention. Cancer Metastasis Rev. 30, 363-385. doi:10.1007/s10555-011-9324-x

Cebola, I., Custodio, J., Muñoz, M., Díez-Villanueva, A., Paré, L., Prieto, P., et al. (2015). Epigenetics Override Pro-inflammatory PTGS Transcriptomic Signature towards Selective Hyperactivation of PGE2 in Colorectal Cancer. Clin. Epigenetics 7, 74. doi:10.1186/s13148-015-0110-4

Doherty, G. A., and Murray, F. E. (2009). Cyclooxygenase as a Target for Chemoprevention in Colorectal Cancer: Lost Cause or a Concept Coming of Age. Expert Opin. Ther. Targets 13, 209-218. doi:10.1517/ 14728220802653631
ER-M: sample preparation, gene expression analysis; LC-T: sample preparation, gene expression analysis; PF: lentivirus preparation and transduction; MT: lentivirus design, experimental design; FC-H: statistics, PCA; AC: experimental design and discussion; MG: gene expression analysis and experimental design; MF: experimental design, funding and article preparation, and discussion.

\section{FUNDING}

This research was funded by grants from "Ministerio de Ciencia e Innovación” (SAF2013-42850-R, SAF2016-75988-R, and PID2019-104760RB-I00), "Comunidad de Madrid (S2017/ BMD-3671. INFLAMUNE-CM), Fondo de Investigaciones Sanitarias" (BIOIMID) to MF and Institutional grants from "Fundación Ramón Areces" and "Banco de Santander". KS was the recipient of a Spanish Association Against Cancer Oncology Investigator grant (AECC AIO).

\section{ACKNOWLEDGMENTS}

We appreciate ANGLE PLC and particularly Michael O’Brien and Clare Halkett for technical assistance and discussions regarding the Parsortix system. We appreciate Carolina Maroto González and Maria Chorro for technical assistance. We appreciate the assistance of the CBMSO Flow Cytometry Service and Animal facility Service usage and personnel assistance.

Garaulet, G., Alfranca, A., Torrente, M., Escolano, A., López-Fontal, R., Hortelano, S., et al. (2013). IL10 Released by a New Inflammation-Regulated Lentiviral System Efficiently Attenuates Zymosan-Induced Arthritis. Mol. Ther. 21, 119-130. doi:10.1038/mt.2012.131

Gervasoni, A., Sandri, M. T., Nascimbeni, R., Zorzino, L., Cassatella, M. C., Baglioni, L., et al. (2011). Comparison of Three Distinct Methods for the Detection of Circulating Tumor Cells in Colorectal Cancer Patients. Oncol. Rep. 25, 1669-1703. doi:10.3892/or.2011.1231

Gkountela, S., Castro-Giner, F., Szczerba, B. M., Vetter, M., Landin, J., Scherrer, R., et al. (2019). Circulating Tumor Cell Clustering Shapes DNA Methylation to Enable Metastasis Seeding. Cell 176, 98-e14. doi:10.1016/j.cell.2018.11.046

Hidalgo-Estévez, A. M., Stamatakis, K., Jiménez-Martínez, M., López-Pérez, R., and Fresno, M. (2020). Cyclooxygenase 2-Regulated Genes an Alternative Avenue to the Development of New Therapeutic Drugs for Colorectal Cancer. Front. Pharmacol. 11, 533. doi:10.3389/fphar.2020.00533

Jiménez-Martínez, M., Ostalé, C. M., van der Burg, L. R., Galán-Martínez, J., Hardwick, J. C. H., López-Pérez, R., et al. (2019). DUSP10 Is a Regulator of YAP1 Activity Promoting Cell Proliferation and Colorectal Cancer Progression. Cancers 11, 1767. doi:10.3390/cancers11111767

Jiménez-Segovia, A., Mota, A., Rojo-Sebastián, A., Barrocal, B., Rynne-Vidal, A., García-Bermejo, M.-L., et al. (2019). Prostaglandin F2a-Induced Prostate Transmembrane Protein, Androgen Induced 1 Mediates Ovarian Cancer Progression Increasing Epithelial Plasticity. Neoplasia 21, 1073-1084. doi:10. 1016/j.neo.2019.10.001

Kawai, N., Tsujii, M., and Tsuji, S. (2002). Cyclooxygenases and colon Cancer. Prostaglandins Other Lipid Mediat 68-69, 187-196. doi:10.1016/s00906980(02)00030-8

Kitz, J., Lowes, L. E., Goodale, D., and Allan, A. L. (2018). Circulating Tumor Cell Analysis in Preclinical Mouse Models of Metastasis. Diagnostics (Basel) 8, 30. doi:10.3390/diagnostics8020030 
Mardia, K. V., Kent, J. T., and Bibby, J. M. (1979). Multivariate Analysis. Academic Press.

Obermayr, E., Agreiter, C., Schuster, E., Fabikan, H., Weinlinger, C., Baluchova, K., et al. (2019). Molecular Characterization of Circulating Tumor Cells Enriched by A Microfluidic Platform in Patients with Small-Cell Lung Cancer. Cells 8, E880. doi:10.3390/cells8080880

Obermayr, E., Maritschnegg, E., Agreiter, C., Pecha, N., Speiser, P., Helmy-Bader, S., et al. (2018). Efficient Leukocyte Depletion by a Novel Microfluidic Platform Enables the Molecular Detection and Characterization of Circulating Tumor Cells. Oncotarget 9, 812-823. doi:10.18632/oncotarget.22549

Patel, S., Issaka, R. B., Chen, E., and Somsouk, M. (2020). Colorectal Cancer Screening and COVID-19. Am. J. Gastroenterol. 116, 433-434. doi:10.14309/ ajg.0000000000000970

Raimondi, C., Nicolazzo, C., Gradilone, A., Giannini, G., De Falco, E., Chimenti, I., et al. (2014). Circulating Tumor Cells: Exploring Intratumor Heterogeneity of Colorectal Cancer. Cancer Biol. Ther. 15, 496-503. doi:10.4161/cbt.28020

Rothwell, P. M., Fowkes, F. G., Belch, J. F., Ogawa, H., Warlow, C. P., and Meade, T. W. (2011). Effect of Daily Aspirin on Long-Term Risk of Death Due to Cancer: Analysis of Individual Patient Data from Randomised Trials. Lancet 377, 31-41. doi:10.1016/S0140-6736(10)62110-1

Rothwell, P. M., Wilson, M., Price, J. F., Belch, J. F., Meade, T. W., and Mehta, Z. (2012). Effect of Daily Aspirin on Risk of Cancer Metastasis: a Study of Incident Cancers during Randomised Controlled Trials. Lancet 379, 1591-1601. doi:10. 1016/S0140-6736(12)60209-8

Sastre, J., Maestro, M. L., Gómez-España, A., Rivera, F., Valladares, M., Massuti, B., et al. (2012). Circulating Tumor Cell Count Is a Prognostic Factor in Metastatic Colorectal Cancer Patients Receiving First-Line Chemotherapy Plus Bevacizumab: a Spanish Cooperative Group for the Treatment of Digestive Tumors Study. Oncologist 17, 947-955. doi:10.1634/theoncologist.2012-0048

Siegel, R. L., Miller, K. D., Goding Sauer, A., Fedewa, S. A., Butterly, L. F., Anderson, J. C., et al. (2020). Colorectal Cancer Statistics, 2020. CA Cancer J. Clin. 70, 145-164. doi:10.3322/caac.21601

Sotelo, M. J., Sastre, J., Maestro, M. L., Veganzones, S., Viéitez, J. M., Alonso, V., et al. (2015). Role of Circulating Tumor Cells as Prognostic Marker in Resected Stage III Colorectal Cancer. Ann. Oncol. 26, 535-541. doi:10.1093/annonc/mdu568

Stamatakis, K., Jimenez-Martinez, M., Jimenez-Segovia, A., Chico-Calero, I., Conde, E., Galán-Martínez, J., et al. (2015). Prostaglandins Induce Early
Growth Response 1 Transcription Factor Mediated Microsomal Prostaglandin E2 Synthase Up-Regulation for Colorectal Cancer Progression. Oncotarget 6, 39941-39959. doi:10.18632/oncotarget.5402

Szczerba, B. M., Castro-Giner, F., Vetter, M., Krol, I., Gkountela, S., Landin, J., et al. (2019). Neutrophils Escort Circulating Tumour Cells to Enable Cell Cycle Progression. Nature 566, 553-557. doi:10.1038/s41586-0190915-y

Tseng, W., Leong, X., and Engleman, E. (2007). Orthotopic Mouse Model of Colorectal Cancer. J. Vis. Exp. 10, e484. doi:10.3791/484

Venables, W. N., and Ripley, B. D. (2002). "Introduction," in Modern Applied Statistics With S Statistics and Computing. Editors W. N. Venables and B. D. Ripley (New York, NY: Springer), 1-12. doi:10.1007/978-0-38721706-2_1

Wang, D., and DuBois, R. N. (2010). The Role of COX-2 in Intestinal Inflammation and Colorectal Cancer. Oncogene 29, 781-788. doi:10.1038/onc.2009.421

Zhang, L., Wang, X., Lai, C., Zhang, H., and Lai, M. (2019). PMEPA1 Induces EMT via a Non-canonical TGF- $\beta$ Signalling in Colorectal Cancer. J. Cel Mol Med 23, 3603-3615. doi:10.1111/jcmm.14261

Conflict of Interest: The authors declare that the research was conducted in the absence of any commercial or financial relationships that could be construed as a potential conflict of interest.

Publisher's Note: All claims expressed in this article are solely those of the authors and do not necessarily represent those of their affiliated organizations, or those of the publisher, the editors and the reviewers. Any product that may be evaluated in this article, or claim that may be made by its manufacturer, is not guaranteed or endorsed by the publisher.

Copyright (c) 2022 Stamatakis, Torres-Gérica, Jiménez-Segovia, Ramos-Muñoz, Crespo-Toro, Fuentes, Toribio, Callejas-Hernández, Carrato, García Bermejo and Fresno. This is an open-access article distributed under the terms of the Creative Commons Attribution License (CC BY). The use, distribution or reproduction in other forums is permitted, provided the original author(s) and the copyright owner(s) are credited and that the original publication in this journal is cited, in accordance with accepted academic practice. No use, distribution or reproduction is permitted which does not comply with these terms. 